\title{
Judgments of other bias in Cochrane systematic reviews of interventions are highly inconsistent and thus hindering use and comparability of evidence
}

3

4

5

6

7 Affiliations:

8 1. Institute of Emergency Medicine in Split-Dalmatia County, Split, Croatia

9 2. Cochrane Croatia, University of Split School of Medicine, Split, Croatia

10 3. Faculty of Medicine and Dentistry, Palacký University Olomouc, Olomouc, Czech

11 Republic

12 4. Medical University of Plovdiv, Plovdiv, Bulgaria

13 5. Faculty of Health Sciences - University of Beira Interior, Covilhã, Portugal

14 6. Agency for Quality and Accreditation in Health Care and Social Welfare, Zagreb, Croatia 15

16

17

$18 *$ Corresponding author:

19 Prof. Livia Puljak, MD, PhD

20

Phone: +385-21-557-807, Fax: +385-21-557-811, E-mail: livia@mefst.hr 


\section{Abstract}

Background: Clinical decisions are made based on Cochrane systematic reviews (CSRs), but implementation of results of evidence syntheses such as CSRs is problematic if the evidence is not prepared consistently. All systematic reviews should assess risk of bias (RoB) in included studies, and in CSRs this is done by using Cochrane RoB tool. However, the tool is not necessarily applied according to the instructions. In this study we aimed to analyze types and judgments of 'other bias' in the RoB tool in CSRs of interventions.

Methods: We analyzed CSRs that included randomized controlled trials (RCTs) and extracted data regarding 'other bias' from the RoB table and accompanying support for the judgment. We categorized different types of other bias.

Results: We analyzed 768 CSRs that included 11369 RCTs. There were 602 (78\%) CSRs that had 'other bias' domain in the RoB tool, and they included a total of 7811 RCTs. In the RoB table of 337 CSRs for at least one of the included trials it was indicated that no other bias was found and supporting explanations were inconsistently judged as low, unclear or high RoB. In the 524 CSRs that described various sources of other bias there were 5762 individual types of explanations which we categorized into 31 groups. The judgments of the same supporting explanations were highly inconsistent. Furthermore, we found numerous other inconsistencies in reporting of sources of other bias in CSRs.

Conclusion: Cochrane authors mention a wide range of sources of other bias in the RoB tool and they inconsistently judge the same supporting explanations. Inconsistency in appraising risk of other bias hinders reliability and comparability of Cochrane systematic reviews.

Furthermore, discrepant and erroneous judgments of bias in evidence synthesis will inevitably hinder implementation of evidence in routine clinical practice and reduce confidence of practitioners in otherwise trustworthy sources of information. 
47 Keywords: systematic review; Cochrane; risk of bias; other bias, inconsistency

Running title: Other bias in Cochrane reviews 


\section{Introduction}

51 Assessment of the risk of bias (RoB) in included studies is an integral part of preparing Cochrane systematic reviews (CSRs). Bias is any systematic error that can negatively affect estimated effects of interventions and lead authors to wrong conclusions about efficacy and safety of analyzed interventions [1].

CSRs use Cochrane's RoB tool, whose aim is to enable better appraisal of evidence and ultimately lead to better healthcare [2]. Cochrane's standard RoB tool has seven domains, of which first six refer to specific potential biases while the seventh domain is called 'other bias', which is used for bias occurring due to any additional problems that were not covered elsewhere in the first six domains [3].

The Cochrane Handbook provides some examples of other potential threats to validity, such as design-specific risk of bias in non-randomized trials, baseline imbalance between groups of participants, blocked randomization in trials that are not blinded, differential diagnostic activity, study changes due to interim results, deviations from the study protocol, giving intervention before randomization, inappropriate administration of an intervention or having co-intervention(s), contamination due to drug pooling among participants, insufficient delivery of intervention, inappropriate inclusion criteria, using instruments that are not sensitive for specific outcomes, selective reporting of subgroups and fraud[3].

This list of potential other sources of bias mentioned in the Cochrane Handbook is limited, and it would therefore be useful to explore potential additional sources of 'other bias'. By consulting a more comprehensive list of potential other biases, systematic review might

71 recognize certain problems in included studies that might not otherwise consider a potential 72 source of bias. 
73 The aim of this study was to analyze the scoring and support for judgment of the category

74 'other bias' in a large number of interventional CSRs of randomized controlled trials (RCTs)

75 published in the Cochrane Database of Systematic Reviews (CDSR). 


\section{Methods}

We conducted a retrospective analysis of published CSRs.

\section{Inclusion and exclusion criteria}

We retrieved CSRs of RCTs about interventions published from July 2015to June 2016 (N = 955) by using Advanced search in The Cochrane Library. Diagnostic CSRs, empty CSRs, overviews of systematic reviews and CSRs withdrawn in this period were excluded. CSRs that included both RCTs and non-randomized trials were included, but only RoB of RCTs was analyzed.

\section{Screening}

One author assessed all titles/abstracts to establish eligibility of CSRs for inclusion. Another author verified the assessments of the first author.

\section{Data extraction and categorization}

Data extraction table was developed and piloted using five CSRs. One author extracted the data and another author verified $10 \%$ of extractions. Of the 77 verified CSRs we found 3

CSRs which were partially extracted (3.9\%), which we consider to be a negligible percentage of discrepancy. We extracted judgments and supporting explanations for judgments from the other bias section of RoB table in CSRs. We also extracted judgments and support for judgments from additional non-standard domains beyond the seven standard RoB domains in RoB table if Cochrane authors used them. For CSRs that did not use the 'other bias' domain in the RoB table or any other additional non-standard domains, we analyzed text of results to 
99

100

101

102

103

104 We analyzed number, type, judgments and inconsistencies for various comments about other

105 risk of bias. We also analyzed characteristics of CSRs where there was no 'other bias' domain

106 for any of the included RCTs, in terms of number and type of additional non-standard RoB

107 domains that were used instead of 'other bias'.

108

109 Statistics

110 We performed descriptive statistics using Microsoft Excel (Microsoft Inc., Redmond, WA,

111 USA). We presented data as frequencies and percentages. In the primary analysis we

112 presented CSRs that had the 'other bias' domain in the RoB table. In the secondary analysis

113 we presented CSRs that did not have the 'other bias' domain, or had different non-standard

114 variations of risk of bias assessment. 


\section{Results}

\section{Primary analysis}

119 We analyzed 768 CSRs that included 11369 RCTs. Among those 768 CSRs, we included in

120 the primary analysis 602 CSRs that had 'other bias' domain in the RoB tables. Those 602

121 CSRs included a total of 7811 RCTs. We analyzed 166 CSRs in the secondary analysis

122 because they either did not have 'other bias' domain in RoB tables ( $N=149)$, or those CSRs

123 had both 'other bias' domain and additional non-standard domains in the RoB tables $(\mathrm{N}=17)$.

124 Out of 602 CSRs in the primary analysis, there were $524(87 \%)$ CSRs that described various

125 sources of bias in the 'other bias' domain, while in $78(13 \%)$ CSRs not a single source of other

126 bias was reported. Furthermore, among 602 CSRs from the primary analysis, there were 337

$127(56 \%)$ CSRs in which at least one included trial indicated that no other bias was found.

128 Terminology for comments about non-existent other bias varied, even within individual

129 CSRs. In 268 (80\%) CSRs only one version of the comment that no other bias was found was

130 used, while in $69(20 \%)$ CSRs Cochrane authors used different expressions in comments to

131 indicate that no other sources of bias were found.

132 In $40(12 \%)$ out of 337 CSRs that indicated that no other bias was found, we observed

133 discrepancies in judgment for this domain. Namely, Cochrane authors in these 40 CSRs

134 sometimes indicated that lack of other bias was associated with low RoB, and sometimes they

135 marked it as unclear or high RoB. In $59(18 \%)$ of these 337 CSRs at least one support for

136 judgment that indicated that no other bias was identified Cochrane authors judged as not

137 being low risk of bias (either high or unclear); in 278 CSRs this was judged as low RoB.

138 In 19 CSRs all comments that referred to no other bias being identified were judged as

139 unclear. In one CSR having no other bias was judged as both low and high. In one CSR the 
same comment was judged in different RCTs as either low or high. In one CSR the same

141 comment was judged in different RCTs as either low, or unclear or high.

142 Of the 7811 trials that were included in the 602 CSRs from the main analysis, in 3703 (47\%)

143 trials domain for other bias indicated in the support for judgment that other bias was not

144 identified. Of those 3703 trials, there were 288 (7.8\%) that were judged as unclear RoB, 4

$145(0.1 \%)$ that were judged as high RoB, while the others $(\mathrm{N}=3411,92.1 \%)$ were judged as low

146 RoB.

148 Sources of other bias

149 In the 524 analyzed CSRs that described various sources of other bias, there were 5762

150 different supporting explanations for judgments of other bias that we categorized into 31

151 categories. In 535 trials it was indicated only that it was not possible to assess other bias. For

$15224(4 \%)$ of those 535 trials it was not indicated why this was not possible, while the most

153 common reasons for not being able to assess other bias were that there were 'insufficient

154 information' $(\mathrm{N}=392,73 \%)$, the trial was published as a conference abstract only $(\mathrm{N}=78$,

$15515 \%$ ) and that the trial was published in foreign language so there were issues with translation

$156(\mathrm{~N}=11,2 \%)$. Cochrane authors were not consistent in judging this type of supporting

157 explanation; for $11(2 \%)$ trials it was judged as high RoB, for $520(94 \%)$ as unclear RoB and

158 for $4(0.7 \%)$ as low RoB.

159 There were 236 trials for which Cochrane authors simply wrote that issues related to other

160 bias were not described or unclear. This type of supporting explanation was also

161 inconsistently judged by the Cochrane authors; 7 (3\%) judged it as low RoB and 229 (97\%) as

162 unclear RoB. 
163 The remaining 4991 explanations for judgments of other bias were divided into 29 categories

164 that are shown in Table 1. The most frequently used categories of explanations for other bias

165 were related to baseline characteristics of participants, funding of a trial, reporting, sample

166 size and conflict of interest (Table 2). Cochrane authors used the domain for other bias to

167 indicate positive, negative and unclear aspects of a trial. For example, three most common

168 types of explanations in the category related to baseline characteristic of participants indicated

169 that either baseline characteristics were similar, or that there was imbalance in baseline

170 characteristics, or that there was insufficient information about it. Among 4991 explanations,

171 we were unable to categorize 85 of them because they were uninformative, including

172 explanations such as 'Adequate' or 'N/A' or 'Other risk of bias was possible'. Finally, there

173 were 112 explanations that were used only once or twice in RoB tables we analyzed so we

174 categorized that group as 'Other explanations'.

175

177 We found 34 CSRs with specific partial data regarding other bias. We divided them into four

178 distinct groups: first group with 28 CSRs that had judgments for 'other bias', but not all had

179 accompanying comments, second group with 4 CSRs where only one included RCT did not

180 have the 'other bias' domain, third group with one CSR with included RCT without 'other

181 bias' domain and included RCT with only judgment without comment, and fourth group with

182 one CSR where RoB table was completely missing for 6 included RCTs. Some CSRs had

183 additional non-standard RoB domains, separately or in addition to the 'other bias' domain.

184 Categories of additional non-standard RoB domains in CSRs are shown in Table 3. 
187 There were 3033 trials for which only one category of explanation was written by Cochrane

188 authors. When the explanation had only one category of comment we could be certain that the

189 judgment referred only to that specific comment so we analyzed those in detail to see how the

190 Cochrane authors judge different explanatory comments. There were 259 types of different

191 explanations among those 3033 trials. We analyzed in more detail those judgments for 20

192 most common explanations of other bias and found very high inconsistency in how Cochrane

193 authors judge the same explanations (Table 2).

\section{Secondary analysis}

Reviews without 'other bias' domain in the RoB table

Among 149 CSRs that did not have 'other bias' domain in the RoB table, there were 102

CSRs that did not have any other replacement domain for 'other bias'. These 102 CSRs used varied number of standard RoB domains. In those 102 CSRs, number of standard RoB

200 domains that were used varied, with one standard RoB domain in 4 CSRs, three RoB domains in 7 CSRs, four RoB domains in 15 CSRs, five domains in 51 CSRs and 6 domains in 25

CSRs.

For this group of CSRs, that did not have the 'other bias' domain in the RoB table, we analyzed texts of results to see whether they mentioned any other sources of bias, beyond the standard six domains, in the section 'Risk of bias in included studies'. We found that 68/102 (67\%) did not mention any sources of other bias in the results of review. However, the remaining $34(33 \%)$ did have comments about the other bias. Three of those 34 stated that

208 they had not found any other risk of bias, while 31 CSRs out of those 34 reported in the text 209 of results that the included studies had had from 1 to 6 different categories of other bias. 
Reviews with both 'other bias' domain and additional non-standard domain(s) for other bias

in RoB tables

213 Nine CSRs had both 'other bias' domain and additional non-standard domain(s) for other bias

214 in RoB tables. Those CSRs used from 1 to 4 additional non-standard domains; 18 in total.

215 Those additional non-standard RoB domains are listed in Table 3 and marked with asterisk.

Reviews without 'other bias' domain but with additional non-standard domain(s)

There were 57 CSRs that did not have the 'other bias' domain, but they did have additional non-standard RoB domains apart from the standard domains in the Cochrane RoB table. Most

of the CSRs had only one additional non-standard domain $(\mathrm{N}=24)$, while others had 2-8 those CSRs without 'other bias' domain.

Reviews that consistently did not use support for judgment or they used non-standard

judgments

We found 9 CSRs that consistently did not use supporting explanations for judgment or they used non-standard judgments. In 5 CSRs authors used judgments low, high or unclear RoB, but without comments as support for judgment. In one CSR all trials were marked with unclear risk of other bias without any comment as support for judgment. In four CSRs all 230 trials were marked with low risk of other bias without any comment as support for judgment.

231 We also found 4 CSRs that did not have judgments low-high-unclear, but different kinds of

232 judgments. One CSR had judgments yes/no without supporting comments; two CSRs had

233 judgments yes, no or unclear, with supporting comments and there was one CSR with

234 judgments A-adequate and B-unclear. 
bioRxiv preprint doi: https://doi.org/10.1101/366591; this version posted July 11, 2018. The copyright holder for this preprint (which was

not certified by peer review) is the author/funder. All rights reserved. No reuse allowed without permission.

235 


\section{Discussion}

237 In this study we analyzed 768 Cochrane systematic reviews, with 11369 included trials. We 238 found that Cochrane authors used numerous different categories of sources of other bias and 239 that they were not judging them consistently. We categorized different types of supporting 240 explanations into 31 categories, and we found numerous other inconsistencies in reporting of 241 sources of other bias in CSRs. Findings of this study are disconcerting because consistency in 242 secondary research is very important to ensure comparability of studies.

243 Insufficient and unclear reporting of the 'other bias' domain was very common in the CSRs

244 we analyzed. Among the most common support for judgment were comments that we

245 categorized as 'not described/unclear', which is puzzling because 'other bias' domain is not 246 specific like the other six domains of the RoB tool, and it is therefore difficult to fathom what 247 it means that other bias was not described or that it was unclear. If the authors did not find 248 sources of other bias, or if they thought that they could not assess other bias because of 249 brevity of report or language issues, they should have stated that. Likewise, for some trials the 250 only supporting explanation was that other bias was 'Adequate'. Without any further 251 explanations, readers cannot know what exactly the Cochrane authors found to be adequate in 252 terms of other potential sources of bias. Many systematic reviews had a high number of 253 included studies, and therefore some comments were repeated multiple times in the same 254 systematic review.

255 The most commonly used specific category of other bias referred to baseline characteristics of 256 participants. In RCTs randomization should ensure allocation of participants into groups that 257 differ only in intervention they received. Randomization should ensure that characteristics of 258 participants that may influence the outcome will be distributed equally across trial arms so 259 that any difference in outcomes can be assumed to be a consequence of intervention 260 [4].Baseline imbalances between the groups may indicate that there was something wrong 
with the randomization process, or that they might be due to chance [5]. Severe baseline imbalances can occur because of deliberate actions of trialists if they aim to intentionally subvert the randomization process [6]or due to unintentional errors.

264 Chance imbalances should not be considered a source of bias, but it may be difficult to distinguish whether baseline imbalances are caused by chance or intentional actions. If there are multiple studies included in a meta-analysis, it could be expected that chance imbalances

267 will act in opposite directions. But the problem may occur if there is a pattern of imbalances across several trials that may favor one intervention over another, suggesting imbalance due to bias and not due to chance [7]. Cochrane is now developing a second generation of the RoB tool, titled RoB 2.0, and one of the signaling questions in the RoB domain about randomization process asks "Were there baseline imbalances that suggest a problem with the 272 randomization process"[7]. The fact that so many Cochrane authors used comments about 273 baseline imbalance as a domain of other bias, and not in the RoB domain about random sequence generation (selection bias) indicate that many Cochrane authors consider that this aspect should be emphasized separately from the selection bias domain.

The second most commonly used category of supporting explanations was related to funding

277 of a trial, and comments about conflicts of interest were the fifth most common category. This

278 is in direct contrast with the recommendations from the Cochrane Handbook, where it is 279 acknowledged that information about vested interests should be collected and presented when 280 relevant, but not in the RoB table; such information should be reported in the table called 281 'Characteristics of included studies' [8]. RoB table should be used to describe specific 282 methodological aspects that may have been influenced by the vested interest and directly lead 283 to RoB [8]. Therefore, it is obvious that the authors of the Cochrane Handbook assume that 284 the influence of sponsors can be mediated via other domains of RoB tool such as selective 285 reporting of favorable outcomes. 
However, Lundh et al. have published a CSR in 2017 about industry sponsorship and research outcomes, in which they included 75 primary studies, which shows that commercial funding leads to more favorable efficacy results and conclusions compared to non-profit funding [9]. They concluded that industry sponsorship introduces bias that cannot be explained by standard domains of Cochrane's RoB assessment [9]. The debate about whether funding

291 presents source of bias or not is ongoing in the Cochrane, with some considering that 292 commercial funding is a clear risk of bias, while others argue against such standpoint[10, 11].

293 This debate apparently reflects the current situation in which many Cochrane authors continue 294 to use funding and conflict of interest as a source of other bias despite the official warning 295 against such use of information about sponsorship from the Cochrane Handbook, as we have 296 demonstrated in this study.

297 The third most frequent category of supporting explanations for other bias was related to poor 298 reporting, where Cochrane authors indicated that relevant information were missing or were 299 inadequately reported. Poor reporting hinders transparency, as it allows authors to avoid 300 attention to weak aspects of their studies. For this reason reporting guidelines should be used $301 \quad[12]$.

302 Comments about sample size were the fourth most common category either in a sense that the 303 trial did or did not report sample size calculation, or that sample size was "small" without any 304 further explanation of what the Cochrane authors considered to be a small sample. There were 30521 trials for which Cochrane authors wrote that there were fewer than 50 participants in each 306 arm. It is unclear where this cut-off is coming from, as there is no such guidance in the 307 Cochrane Handbook in the chapter about risk of bias. On the contrary, chapter 8.15.2. of the 308 Cochrane Handbook specifically warns that "sample size or use of a sample size (or power) 309 calculation“ are examples of quality indicators that ,should not be assessed within this 310 domain“"[8]. 
311 The Cochrane Handbook also warns that authors should avoid double-counting, by not

312 including potential sources of bias in the 'other bias' domain if they can be more appropriately

313 covered by other domains in the tool [8]. As can be seen by our study, Cochrane authors

314 sometimes do double-counting because there were categories of comments supporting

315 judgments that could have been addressed in the first six domains.

316 As we have shown, most of Cochrane authors decided to use the other bias domain to describe

317 potential additional biases that were not covered in the first six domains of the RoB tool. In

318 the proposed RoB tool 2.0 there is no 'other bias' domain [7]. The proposed RoB tool is much

319 more complex, compared to the current version of the RoB tool, and many items that were

320 specifically emphasized by Cochrane authors in the other bias domain, as shown in our study,

321 are addressed in the RoB 2.0 tool. However, there are still potential biases from other sources

322 that the RoB 2.0 may neglect by omitting the RoB domain, such as biases specific to certain

323 topics, and those that were not recognized by the RoB 2.0 tool in advance.

324 We have already conducted a similar analysis of Cochrane RoB domain related to attrition

325 bias, and we found that judgments and supports for judgments in that domain were extremely

326 inconsistent in CSRs (unpublished data). This analysis related to sources of other bias in

327 CSRs contributes to the perception that Cochrane RoB tool is inconsistently used among

328 Cochrane authors. The authors do not necessarily follow guidance from the Cochrane

329 Handbook. In the support for judgment they mention issues that the Cochrane Handbook

330 explicitly warns against. Various comments that serve as supports for judgments were

331 inconsistently judged across CSRs and trials included in CSRs. Cochrane authors also use

332 inconsistent terminology to describe the same concepts. Increasing complexity of the RoB

333 tool, as proposed in the RoB tool 2.0 will likely only increase this problem of insufficient

334 consistency in RoB appraisal and worsen this problem of insufficient comparability of

335 judgments of RoB across CSRs. 
336 Furthermore, our study indicated that Cochrane authors extensively use the available option to customize the RoB table. We found that there were as many as $102(13 \%)$ out of 768 analyzed

338 CSRs that did not use the other bias domain in the RoB table at all. CSRs are produced using

339 software Review Manager (RevMan). As soon as an author inserts a new study in the

340 RevMan among included studies, an empty RoB table for the study automatically appears,

341 with seven pre-determined domains. Therefore, Cochrane authors need to intentionally

342 remove or add some domains if they want to customize the RoB table. Among 102 CSRs that

343 did not have other bias domain, 33\% of those CSRs had comments about other potential

344 sources of bias in the body of the manuscript. It is unclear why some Cochrane authors use

345 only text for comments about other bias instead of using RoB table for this purpose.

346 Additionally, we observed that in many CSRs without other bias domain there were other

347 customizations of the RoB table, which had from one to six other, standard RoB domains

348 included. Exactly half of those CSRs without other bias domain in the RoB table had less than

349 six standard domains in the RoB table.

350 Limitation of our study is a limited number of analyzed CSRs that were published in 2015 and

351 2016. However, considering the number of CSRs analyzed, and the amount of inconsistency

352 we observed, we have no reason to suspect that the results would be significantly different if a

353 more recent cohort of published CSRs would have been used. Additionally, it takes a long

354 time to categorize thousands of different inconsistent supporting explanations. Some

355 unintentional errors in categorizations may have been made. 


\section{Conclusion}

358 Cochrane authors mention a wide range of sources of other bias in the RoB tool and they

359 inconsistently judge the same supporting explanations. Inconsistency in appraising risk of

360 other bias hinders reliability and comparability of Cochrane systematic reviews. Discrepant

361 and erroneous judgments of bias in evidence synthesis will inevitably hinder implementation

362 of evidence in routine clinical practice and reduce confidence of practitioners in otherwise

363 trustworthy sources of information. Potential remedies include more attention to author

364 training, better resources for Cochrane authors, better peer-review and editorial consistency in

365 the production of Cochrane systematic reviews. 


\section{Declarations}

Ethics approval and consent to participate

369 Not applicable; this was secondary study

Consent for publication

372 Not applicable

Availability of data and material

375 The datasets used and/or analysed during the current study are available from the corresponding author on reasonable request.

\section{Competing interests}

The authors declare that they have no competing interests.

Funding

382 No extramural funding.

Authors' contributions

385 Study design: LP. Data analysis and interpretation: AB, AP, LB, YG, MARP, TPP, LP.

386 Drafting the first version of the manuscript: AB, LP. Revisions of the manuscript: AB, AP,

387 LB, YG, MARP, TPP, LP. All authors read and approved the final manuscript. All authors 388 agree to be accountable for this work.

391 We are grateful to Ms. Dalibora Behmen for language editing. 


\section{References}

393 1. Gluud LL: Bias in clinical intervention research. Am J Epidemiol 2006, 163(6):493394501.

395 2. Higgins JP, Altman DG, Gotzsche PC, Juni P, Moher D, Oxman AD, Savovic J, Schulz KF, Weeks L, Sterne JA: The Cochrane Collaboration's tool for assessing risk of bias in randomised trials. BMJ 2011, 343:d5928.

3. Higgins JPT, Green Se: Cochrane Handbook for Systematic Reviews of Interventions Version 5.1.0 [updated March 2011]. The Cochrane Collaboration. Available from www.cochrane-handbook.org. In.; 2011.

401

4. Roberts C, Torgerson DJ: Understanding controlled trials - Baseline imbalance in randomised controlled trials. Br Med J 1999, 319(7203):185-185.

5. Fu R, Vandermeer BW, Shamliyan TA, O'Neil ME, Yazdi F, Fox SH: AHRQ Methods for Effective Health Care: Handling Continuous Outcomes in Quantitative Synthesis. Methods Guide for Effectiveness and Comparative Effectiveness Reviews. Rockville (MD): Agency for Healthcare Research and Quality (US). 2008.

6. Schulz KF: Subverting Randomization in Controlled Trials. Jama-J Am Med Assoc 1995, 274(18):1456-1458.

7. A revised tool to assess risk of bias in randomized trials (RoB 2.0). Available at: https://sites.google.com/site/riskofbiastool//welcome/rob-2-0-tool.

411 8. Higgins J: Chapter 8: assessing risk of bias in included studies. In: Higgins J, Green S, editors. Cochrane Handbook for Systematic Reviews of Interventions Version 510 (updated March 2011), The Cochrane Collaboration; 2011 [Available from www.cochrane-handbook.org]. Last accessed: February 17, 2015. 2011.

$4159 . \quad$ Lundh A, Lexchin J, Mintzes B, Schroll JB, Bero L: Industry sponsorship and 416 research outcome. Cochrane Database Syst Rev 2017, 2:MR000033. 
417 10. Bero LA: Why the Cochrane Risk of Bias Tool Should Include Funding Source as a

$418 \quad$ Standard Item. Cochrane Database of Systematic Reviews 2013(12).

419 11. Sterne JAC: Why the Cochrane Risk of Bias Tool Should Not Include Funding Source as a Standard Item. Cochrane Database of Systematic Reviews 2013(12).

421 12. Schulz KF, Altman DG, Moher D: CONSORT 2010 Statement: updated guidelines for reporting parallel group randomised trials. Bmc Med 2010, 8:18.

423

424 
bioRxiv preprint doi: https://doi.org/10.1101/366591; this version posted July 11,2018 . The copyright holder for this preprint (which was not certified by peer review) is the author/funder. All rights reserved. No reuse allowed without permission. 
Table 1. Different categories of other bias (based on 4991 explanations) in Cochrane systematic reviews

\section{Category}

Baseline characteristics of participants

$\mathbf{N}(\%)$

1067 (21.4)

Funding

$774(15.6)$

Sample size

$405(8.1)$

Reporting

Conflict of interest

Inclusion and exclusion criteria

Confounding

Analyses

Outcome domains and outcome measures

Co-interventions

Deviations from the protocol

Randomisation

Terminated early

Issues related to cross-over trials

Intention-to-treat analysis (ITT)

Study design

Compliance

Attrition

Contamination

Follow-up and study duration

Blinding

Clustering

Selection bias

Protocol registration

Study quality

Publication bias

Adequacy of comparators

Inexplicable

Other
$381(7.6)$

$288(5.8)$

$197(3.9)$

$196(3.9)$

$191(3.8)$

$135(2.7)$

$134(2.7)$

$123(2.5)$

$111(2.2)$

108 (2.2)

$98(2)$

95 (1.9)

76 (1.6)

$72(1.4)$

71 (1.4)

65 (1.3)

$46(0.9)$

$25(0.5)$

$17(0.3)$

$17(0.3)$

$16(0.3)$

$9(0.2)$

$7(0.1)$

$5(0.1)$

85 (1.7)

177 (3.6) 
431 Table 2. Judgments for the 20 most common explanations of other bias

\begin{tabular}{|c|c|c|c|c|}
\hline Explanation & Total & High, N (\%); n* & $\begin{array}{r}\text { Unclear, } \mathbf{N} \\
(\%) ; \mathbf{n}^{*}\end{array}$ & $\begin{array}{l}\text { Low, N (\%); } \\
\text { n* }^{*}\end{array}$ \\
\hline $\begin{array}{l}\text { Not possible to assess other } \\
\text { bias }\end{array}$ & 504 & $7(1.4) ; 7$ & $494(98) ; 117$ & $3(0.6) ; 3$ \\
\hline $\begin{array}{l}\text { Baseline characteristics similar } \\
\text { between the groups }\end{array}$ & 314 & $0(0) ; 0$ & $24(8) ; 13$ & $290(92) ; 61$ \\
\hline Not described/unclear & 233 & $0(0) ; 0$ & $226(97) ; 54$ & $7(3) ; 4$ \\
\hline $\begin{array}{l}\text { Baseline imbalance between } \\
\text { groups of participants }\end{array}$ & 167 & $91(54) ; 56$ & $62(37) ; 41$ & $14(9) ; 12$ \\
\hline Funding: industry & 162 & $83(51) ; 28$ & $77(48) ; 25$ & $2(1) ; 2$ \\
\hline Potential confounding factors & 120 & $63(53) ; 38$ & $47(39) ; 34$ & $10(8) ; 9$ \\
\hline $\begin{array}{l}\text { Not enough information on } \\
\text { baseline characteristics of } \\
\text { participants }\end{array}$ & 88 & $8(9) ; 6$ & $78(89) ; 39$ & $2(2) ; 2$ \\
\hline Funding: non-profit & 86 & $0(0) ; 0$ & $4(5) ; 4$ & $82(95) ; 33$ \\
\hline Funding: not reported & 72 & $0(0) ; 0$ & $68(94) ; 15$ & $4(6) ; 4$ \\
\hline $\begin{array}{l}\text { Important parameters not } \\
\text { reported }\end{array}$ & 61 & $19(31) ; 14$ & $41(68) ; 28$ & $1(1) ; 1$ \\
\hline $\begin{array}{l}\text { Sample size: calculation of } \\
\text { sample size not provided }\end{array}$ & 42 & $24(57) ; 6$ & $17(41) ; 7$ & $1(2) ; 1$ \\
\hline $\begin{array}{l}\text { Potential randomisation } \\
\text { problem }\end{array}$ & 40 & $9(23) ; 9$ & $28(70) ; 13$ & $3(7) ; 3$ \\
\hline $\begin{array}{l}\text { Potential problem with } \\
\text { inclusion criteria }\end{array}$ & 40 & $16(40) ; 15$ & $22(55) ; 12$ & $2(5) ; 2$ \\
\hline $\begin{array}{l}\text { Deviations from the study } \\
\text { protocol }\end{array}$ & 37 & $\begin{array}{r}16(43) \\
13 \\
\end{array}$ & $\begin{array}{r}18(49) \\
15 \\
\end{array}$ & $\begin{array}{r}3(8) \\
3 \\
\end{array}$ \\
\hline No relevant subgroup analysis & 36 & $10(28) ; 1$ & $26(72) ; 1$ & $0(0) ; 0$ \\
\hline $\begin{array}{l}\text { Funding: intervention supplied } \\
\text { by industry }\end{array}$ & 32 & $14(44) ; 7$ & $12(38) ; 10$ & $6(18) ; 3$ \\
\hline Adequate & 28 & $0(0) ; 0$ & $0(0) ; 0$ & $28(100) ; 1$ \\
\hline $\begin{array}{l}\text { No information on the validity } \\
\text { of the outcome measure }\end{array}$ & 27 & $3(11) ; 3$ & $23(85) ; 5$ & $1(4) ; 1$ \\
\hline $\begin{array}{l}\text { Sample size: performed } \\
\text { calculation }\end{array}$ & 24 & $1(4) ; 1$ & $3(12) ; 3$ & $20(84) ; 9$ \\
\hline Sample size: small & 23 & $8(35) ; 5$ & $15(65) ; 5$ & $0(0) ; 0$ \\
\hline
\end{tabular}


Table 3. Categories of additional non-standard RoB domains in Cochrane systematic reviews

\begin{tabular}{|c|c|}
\hline Additional category & N of CSRs \\
\hline Group similarity at baseline (selection bias) & 11 \\
\hline Baseline data & 5 \\
\hline Baseline outcome measures (similar) & 3 \\
\hline Groups balanced at baseline/ balance in baseline characteristics & 2 \\
\hline Baseline characteristics of participants & 1 \\
\hline Baseline comparability of treatment and control groups & 1 \\
\hline Baseline measures & 1 \\
\hline Similarity of baseline characteristics* & 1 \\
\hline Treatment/control groups comparative at entry & 1 \\
\hline Major imbalance in important baseline confounders & 1 \\
\hline $\begin{array}{l}\text { Comparability of groups on different prognostic } \\
\text { characteristics* }\end{array}$ & 1 \\
\hline Size & 8 \\
\hline Size of the study & 5 \\
\hline Small sample size bias & 4 \\
\hline Sample size* & 2 \\
\hline Sufficient sample size* & 1 \\
\hline Power calculation* & 1 \\
\hline Timing of outcome assessment (similar)* & 10 \\
\hline Adequate follow-up & 2 \\
\hline Study duration & 2 \\
\hline Early stopping & 1 \\
\hline Groups received comparable treatment & 2 \\
\hline Care program identical/ identical care & 2 \\
\hline Treatment fidelity* & 1 \\
\hline Free of systematic differences in care?* & 1 \\
\hline Consistency in intervention delivery & 1 \\
\hline Equality of treatment & 1 \\
\hline Protocol deviation balanced & 1 \\
\hline Groups received same intervention & 1 \\
\hline Compliance/adherence assessed (acceptable) & 7 \\
\hline Compliance with recommendation reliable? & 1 \\
\hline Compliance acceptable* & 1 \\
\hline Source of funding/ sponsorship & 4 \\
\hline For profit funding* & 1 \\
\hline Funding* & 1 \\
\hline Vested interest bias & 1 \\
\hline Conflict of interest & 1 \\
\hline Co-intervention avoided or similar* & 5 \\
\hline Co-interventions & 2 \\
\hline Groups received same co- interventions & 1 \\
\hline Intention to treat & 5 \\
\hline Incorrect analysis & 1 \\
\hline Results based on data dredging? & 1 \\
\hline Analyses adjust for different lengths of follow-up workers? & 1 \\
\hline Appropriate statistical tests use? & 1 \\
\hline
\end{tabular}




\begin{tabular}{|l|l|}
\hline Adequate adjustment for confounding in the analyses? & 1 \\
\hline Contamination/ protection against contamination & 3 \\
\hline Validity of outcome measures & 1 \\
Reliability of outcome measures & 1 \\
Outcome measures used valid and reliable? & 1 \\
\hline $\begin{array}{l}\text { Free from performance bias } \\
\text { Performance bias as «differential expertise» bias }\end{array}$ & 1 \\
$\begin{array}{l}\text { Performance bias as comparability in the experience of care } \\
\text { providers }\end{array}$ & 1 \\
\hline $\begin{array}{l}\text { Adequate patient description } \\
\text { Recruitment of participants from the same population? }\end{array}$ & 1 \\
Recruitment of participants over the same study period? & 1 \\
\hline Washout/ carry-over effect in cross-over study designs & 1 \\
\hline Overall assessment of bias risk & 2 \\
\hline Summary of risk of bias for Consumption outcome & 1 \\
\hline Researcher allegiance* & 1 \\
\hline Therapist allegiance* & 1 \\
\hline CHBG (Cochrane hepato-biliary group) combined assessment & 1 \\
\hline (mortality)* & 1 \\
\hline CHBG combined assessment (hepatic encephalopathy)* & 1 \\
\hline Detection bias (biochemical validation of smoking outcomes) & 1 \\
\hline Ethical approval & 1 \\
\hline Explicit inclusion/exclusion criteria & 1 \\
\hline Free of dietary differences other than fat?* & 1 \\
\hline Loss of clusters & 1 \\
\hline Methods for selecting cases to adjudicate & 1 \\
\hline Outcome description & 1 \\
\hline Publication format & 1 \\
\hline Recruitment bias & 1 \\
\hline
\end{tabular}

436 *domains found in 9 Cochrane reviews that had both 'other bias' domain and additional non437 standard domain(s) for other bias in RoB tables 